\title{
Fractional-Order Sliding Mode Control of Overhead Cranes
}

\author{
Pham Van Trieu, Pham Duc Toan, Hoang Manh Cuong* \\ Vietnam Maritime University, Haiphong, Vietnam \\ *Email: cuonghm@vimaru.edu.vn
}

\begin{abstract}
We constitute a control system for overhead crane with simultaneous motion of trolley and payload hoist to destinations and suppression of payload swing. Controller core made by sliding mode control (SMC) assures the robustness. This control structure is inflexible since using fixed gains. For overcoming this weakness, we integrate variable fractional-order derivative into SMC that leads to an adaptive system with adjustable parameters. We use Mittag-Leffler stability, an enhanced version of Lyapunov theory, to analyze the convergence of closed-loop system. Applying the controller to a practical crane shows the efficiency of proposed control approach. The controller works well and keeps the output responses consistent despite the large variation of crane parameters.
\end{abstract}

Keywords: Fractional-order control, overhead cranes, sliding mode control.

\section{Introduction}

Using frequently in industrial transportation, an overhead crane is equipped with three mechanisms for lifting and transferring material and package in factories. The modern cranes speedy run or/and combine the motions for increasing productivity. This causes imprecise motions and large swing of payload if cranes do not have suitable control strategies. Many control algorithms were proposed for overhead cranes [1-18]. The articles [1-12] in connection to control of overhead cranes were published from linear control [1], feedback linearization [2], pole-placement [3], linear quadratic optimal control [4], to complicated nonlinear control [5], model predict control [6], adaptive control approach [7-8], robust control methods [9-10], and modern control techniques such as fuzzy logic [11], neural networks [12]. Focusing on robust controls, SMC [13] is a control approach which works effectively for cranes. SMC does not require much the modelling precision. It may work well with un-modeled dynamics. Additionally, it shows the robustness when the system faces disturbances and uncertainties. Its advanced versions such as terminal SMC [14], fast terminal SMC [15] make quick finite-time convergence. SMC can combine with fuzzy [16], neural network [17], selftuning control [18] to achieve both robust and adaptive features. Accordingly, adaptive mechanisms are supplemented for estimating uncertainties, unknown factors, and disturbances. On the upside, fractional calculus has been widely applied to control engineering in the recent years [22]. Instead of using fixed first/second-order derivatives for feedback signals, fractional-derivative (FD) makes flexible control structure in which it is tunable to get optimal

ISSN: 2734-9373

https://doi.org/10.51316/jst.150.ssad.2021.31.1.9

Received: 12 January 2021; accepted: 11 March 2021 performance. This study utilizes strong points of both SMC and FD. We create a flexibly robust controller by integrating FD into SMC core. The controller tracks trolley and hoist payload to desired positions, concurrently keeps the payload swing small at transient state, and suppresses this swing at steady state. Such the combination applied for overhead cranes has not been has not been released until recently. Proposed control system shows the following advantages:

(i) The controller well immunizes disturbances and is robust with uncertainties due to the action of SMC structures.

(ii) Due to using variable FD, control structure may vary flexibility to adapt with uncertain environment. Tuning FD orders may get the optimal system responses.

In fact, control formulation of cranes is classified into low-level control (LLC) and high-level planning (HLP). LLC deals with precise tracking trolley and crane to destinations while keeping payload swing small and vanishing at steady-state. HLP develops algorithms for motion planning and trajectories to prevent the obstacles. At modern crane, two control options may be combined.

This study focuses on LLC. We organize the article as follows. Section 2 provides concept of fractional calculus [7] and stability theory for fractional systems [8-10]. Section 3 describes physical features of an overhead crane through its dynamic model. Section 4 designs controller by combining SMC with FD, analyzing crane stability with Mittag-Leffler sense [9] is also included. Section 5 tests the control algorithms on a practical overhead crane, analyzes and discusses the application results. Finally, several conclusions and remarks are represented in Section 6. 


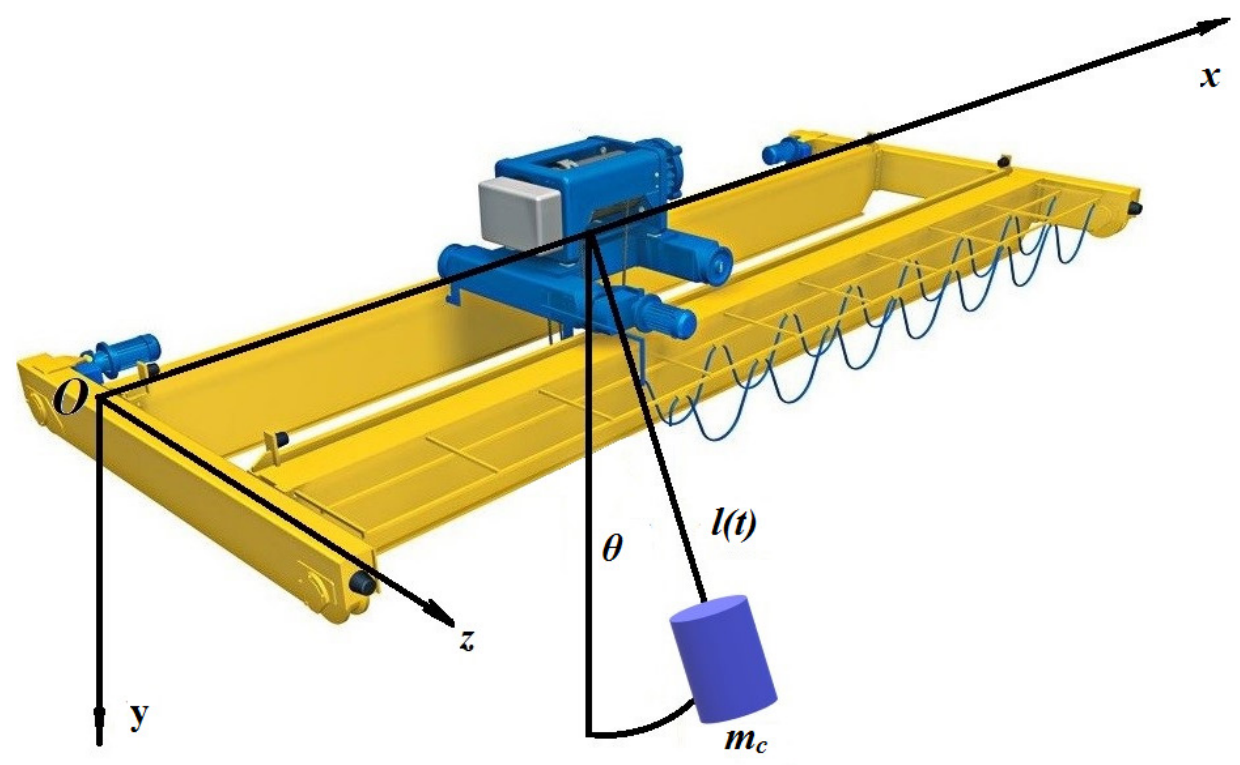

Fig. 1. 2D motion of an overhead crane.

\section{Fractional Derivative in Control}

We utilize the concept of fractional calculus and related topic to design control system. The following definition, theorem, and lemma will be applied for analyzing and constituting the control algorithm at the next sections:

Definition 1 [19]: Fractional-order $\alpha$ derivative of function $f(t)$ with time defined by Caputo is given as

$$
D_{t}^{\alpha} f(t)=\frac{1}{\Gamma(m-\alpha)} \int_{0}^{t}(t-\tau)^{m-\alpha-1} g(\tau) \mathrm{d} \tau
$$

with $0<\alpha<1, \quad m \in \mathrm{Z}^{+}$and $\Gamma(z)=\int_{0}^{\infty} e^{-t} t^{z-1} \mathrm{~d} t \quad$ being gamma function.

Lemma 1 [20]: Let $\mathbf{x}(t) \in R^{n}$ be a state vector. The inequality

$$
0.5 D_{t}^{\alpha}\left(\mathbf{x}^{T} \mathbf{Q} \mathbf{x}\right) \leq \mathbf{x}^{T} \mathbf{Q} D_{t}^{\alpha} \mathbf{x}
$$

is held for every positive definite matrix $\mathbf{Q} \in R^{n \times n}$.

Theorem 1 [21]: If existing a continuously differentiable function $V(\mathbf{x}, t)$ satisfying

$$
\alpha_{1}\|\mathbf{x}\|^{a} \leq V(\mathbf{x}, t) \leq \alpha_{2}\|\mathbf{x}\|^{a b}
$$

and

$$
D_{t}^{\alpha} V(\mathbf{x}, t) \leq-\alpha_{3}\|\mathbf{x}\|^{a b},
$$

then $\mathbf{x}=\mathbf{0}$ is global stability in the sense of MittagLeffler. Here, $a, b, \alpha_{1}, \alpha_{2}$, and $\alpha_{3}$ are positive constants.
Theorem 2 [22]: Fractional LTI system

$$
D_{t}^{\alpha} \mathbf{x}(t)=\mathbf{A x}(t)
$$

with $\mathbf{A} \in R^{n \times n}$ is stable if it justifies

$$
|\arg (\operatorname{eig} \mathbf{A})|>\alpha \pi / 2
$$

\section{Dynamical Description}

We consider simultaneous motion of trolley $m_{t}$ and payload hoist $m_{l}$ when operating an overhead crane as in Fig. 1. Three outputs composed of moving trolley $x$, lifting (with varying cable length $l$ ) payload $m_{c}$, and swinging $\theta$ payload are controlled by two inputs: $u_{t}$ is trolley-pushing force and $u_{l}$ is payload lifting force. $b_{t}$ and $b_{r}$ are parameters for frictions at trolley and cable.

Dynamic behavior of cranes is governed by actuated model:

$$
\begin{aligned}
& \left(m_{t}+m_{c}\right) \ddot{x}-m_{c} \sin \theta \ddot{l}-m_{c} l \cos \theta \ddot{\theta} \\
& +b_{t} \dot{x}-2 m_{c} \cos \theta i \dot{\theta}+m_{c} l \sin \theta \dot{\theta}^{2}=u_{t}(t) \\
& -m_{c} \sin \theta \ddot{x}+\left(m_{c}+m_{l}\right) \ddot{l}+b_{r} l \\
& -m_{c} l \dot{\theta}^{2}-m_{c} g \cos \theta=u_{l}(t)
\end{aligned}
$$

corresponding $\mathbf{q}_{a}=[x l]^{T}$, and swinging equation

$$
\ddot{\theta}=(1 / l)(\cos \theta \ddot{x}-2 i \dot{\theta}-g \sin \theta)
$$

describes payload swing $\theta$. Substituting Eq. (9) into Eqs. (7), (8) and rearranging leads to a reduced-order dynamics

$$
\overline{\mathbf{M}} \ddot{\mathbf{q}}_{a}+\mathbf{B} \dot{\mathbf{q}}_{a}+\mathbf{f}(\mathbf{q}, \dot{\mathbf{q}})=\mathbf{U}
$$




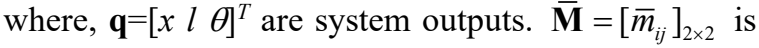
a symmetric matrix with $\bar{m}_{11}=m_{t}+m_{c} \sin ^{2} \theta, \bar{m}_{12}=\bar{m}_{21}=-m_{c} \sin \theta, \quad$ and $\bar{m}_{22}=m_{c}+m_{l} . \quad \mathbf{B}=\operatorname{diag}\left(b_{t}, b_{r}\right) \quad$ denotes damping. $\mathbf{f}(\mathbf{q}, \dot{\mathbf{q}})=\left[\left(m_{c} l \sin \theta \dot{\theta}^{2}+0.5 m_{c} g \sin 2 \theta\right)-\left(m_{c} \dot{\theta}^{2}+m_{c} g \cos \theta\right)\right]^{T}$, and $\mathbf{U}=\left[\begin{array}{ll}u_{t} & u_{l}\end{array}\right]^{T}$ is outputs.

\section{Controller Design}

We design a structure for tracking $\mathbf{q}_{a}$ to destination $\mathbf{q}_{a d}=\left[\begin{array}{ll}x & l\end{array}\right]^{T}$ while suppressing payload swing ( $\theta$ reaches zero). We consider a sliding manifold containing fractional derivative

$$
\mathbf{s}=D_{t}^{\alpha}\left(\mathbf{q}_{a}-\mathbf{q}_{a d}\right)+\lambda\left(\mathbf{q}_{a}-\mathbf{q}_{a d}\right)+\boldsymbol{\beta} \theta
$$

where, $0 \leq \alpha \leq 1$ is fractional-order (FO) of Caputo derivative (Definition 1), $\boldsymbol{\lambda}=\operatorname{diag}\left(\lambda_{1}, \lambda_{2}\right)$ is a positive matrix, and $\boldsymbol{\beta}=\left[\begin{array}{ll}\beta_{1} & 0\end{array}\right]^{T}$. A control law is proposed compatible with surface (11) by a following statement.

Statement: A structure of fractional sliding mode control

$$
\begin{aligned}
\mathbf{U}= & -\overline{\mathbf{M}} D_{t}^{(2-\alpha)}\left\{\boldsymbol{\lambda}\left(\mathbf{q}_{a}-\mathbf{q}_{a d}\right)+\boldsymbol{\beta} \theta\right\} \\
& +\mathbf{B} \dot{\mathbf{q}}_{a}+\mathbf{f}(\mathbf{q}, \dot{\mathbf{q}})-\boldsymbol{\eta} \operatorname{sgn} \mathbf{s}
\end{aligned}
$$

tracks outputs q governed by crane dynamics (7)-(9) to destinations $\mathbf{q}_{d}=\left[\begin{array}{lll}x_{d} & l_{d} & 0\end{array}\right]^{T}$ asymptotically. Here, $\boldsymbol{\eta}=\operatorname{diag}\left(\eta_{1}, \eta_{2}\right)$ are two positive gains. function

Proof: We begin with a bounded Lyapunov

$$
V=0.5 \mathbf{s}^{T} \overline{\mathbf{M}} \mathbf{s}>0
$$

Since $\quad \bar{m}_{11}=\left(m_{t}+m_{c} \sin ^{2} \theta\right)>0 \quad$ and $\operatorname{det} \overline{\mathbf{M}}=\left[m_{t}\left(m_{c}+m_{l}\right)+m_{c} m_{l} \sin ^{2} \theta\right]>0, \quad \overline{\mathbf{M}} \quad$ is positive definite. Based on Lemma 1, fractional derivative of Lyapunov (13) satisfies

$$
\begin{aligned}
& D_{t}^{\alpha} V \leq \mathbf{s}^{T} \overline{\mathbf{M}} D_{t}^{\alpha} \mathbf{s} \\
& =\mathbf{s}^{T} \overline{\mathbf{M}}\left[D_{t}^{2(\alpha-1)} \ddot{\mathbf{q}}_{a}+\lambda D_{t}^{\alpha}\left(\mathbf{q}_{a}-\mathbf{q}_{a d}\right)+\boldsymbol{\beta} D_{t}^{\alpha} \theta\right]
\end{aligned}
$$

Submitting equivalent dynamics (10) and controller (12) into Eq. (14) yields

$$
D_{t}^{\alpha} V \leq \mathbf{s}^{T} \overline{\mathbf{M}} D_{t}^{\alpha} \mathbf{s}=-\mathbf{s}^{T} \boldsymbol{\eta} D_{t}^{2(\alpha-1)} \operatorname{sgn} \mathbf{s}
$$

For $\alpha \neq 1$, Caputo derivative $D_{t}^{2(\alpha-1)} \operatorname{sgn} \mathbf{s}=0$ leads inequality (15) to

$$
D_{t}^{\alpha} V \leq 0
$$

The case $\alpha=1$ leads inequality (15) to

$$
D_{t}^{\alpha} V \leq-\eta_{1}\left|s_{1}\right|-\eta_{2}\left|s_{2}\right|
$$

The expressions (13), (16), and (17) fit conditions of Theorem 1. This implies that equilibrium $\mathbf{s}=\mathbf{0}$ is globally stable. The convergence of manifold (11) produces a linear fractional-order system

$$
D_{t}^{\alpha}\left(\mathbf{q}_{a}-\mathbf{q}_{a d}\right)=-\lambda\left(\mathbf{q}_{a}-\mathbf{q}_{a d}\right)-\boldsymbol{\beta} \theta
$$

Physically, $\theta$ is always toward 0 due to payload weight even without control, and gain $\boldsymbol{\beta}$ supports the fast convergence of $\theta$. Eq. (18) is reduced as

$$
D_{t}^{\alpha}\left(\mathbf{q}_{a}-\mathbf{q}_{a d}\right)=-\lambda\left(\mathbf{q}_{a}-\mathbf{q}_{a d}\right)
$$

Applying Theorem 2 to system (19) indicates that $\left(\mathbf{q}_{a}-\mathbf{q}_{a d}\right)$ is locally stabile around $\mathbf{0}$ for every positive definite matrix $\boldsymbol{\lambda}$. Thus, tracking $\mathbf{q}_{a}$ to $\mathbf{q}_{a d}$ is achieved.

Remark 1: Sign function of control law (12) may cause the chattering at system responses. There are several ways to reduce this, such as: replacing sign action by situation function or sigmoid function, using a filter or estimator, higher-order solution, super-twisting method, and so on. In this article, we utilize a hyperbolic tangent function

$$
\tanh \mathbf{s}=\left[\tanh s_{i}\right]_{i}=\left[\frac{e^{s_{i}}-e^{-s_{i}}}{e^{s_{i}}+e^{-s_{i}}}\right]_{i}
$$

replacing for sgns function.

Remark 2: Theoretically, sliding surface (11) of controller (12) only assures the infinity convergence. In fact, the control law (12) is designed based on the infinity stability of the dynamics

$$
D^{\alpha} \mathbf{s}+\boldsymbol{\eta} \operatorname{sgn} \mathbf{s}=\mathbf{0}
$$

An enhanced version $[10,14,15]$ of SMC, that is the so-called Terminal SMC (TSMC), guarantees the finite-time stability of system outputs. By improving the dynamics of sliding surface as

$$
D^{\alpha} \mathbf{s}+\boldsymbol{\lambda} \mathbf{s}^{q / p}+\boldsymbol{\eta} \operatorname{sgn} \mathbf{s}=\mathbf{0}
$$

with $\eta=\operatorname{diag}\left(\eta_{1}, \eta_{2}\right)$ being positive diagonal matrix, $q$ and $p$ being positive odd integers satisfying $q>p$, we can obtain the TSMC controller in which the terminal stability of sliding manifold is held.

\section{Results and Analysis}

We check the quality of proposed controller (12) on crane dynamics (7)-(9) using a laboratory overhead crane whose parameters: $m_{t}=5 \mathrm{~kg}, m_{c}=0.85$ $\mathrm{kg}, m_{l}=2 \mathrm{~kg}, b_{t}=20 \mathrm{Ns} / \mathrm{m}, b_{r}=\mathrm{Ns} / \mathrm{m}$, and FO-SMC gains: $\boldsymbol{\lambda}=\operatorname{diag}(0.7,0.9), \boldsymbol{\beta}=\left[\begin{array}{ll}1.2 & 0\end{array}\right]^{T}, \boldsymbol{\eta}=\operatorname{diag}(100,100)$. The initial conditions: $\mathbf{q}(0)=\left[\begin{array}{lll}0 & 0.1 & 0\end{array}\right]^{T}$ and $\dot{\mathbf{q}}(0)=\mathbf{0}$. We use three cases of fractional order $\alpha=0.8,0.9$, and 1 . The references of trolley and payload hoisting respectively compose of destinations: $x_{d}=\left[\begin{array}{lll}0 & 0.3 & 0.1\end{array}\right.$ $0.7] \mathrm{m}$ and $l_{d}=\left[\begin{array}{llll}0.1 & 0.4 & 0.6 & 0.2\end{array}\right] \mathrm{m}$. The crane performances are depicted in Figs. 2-6. 


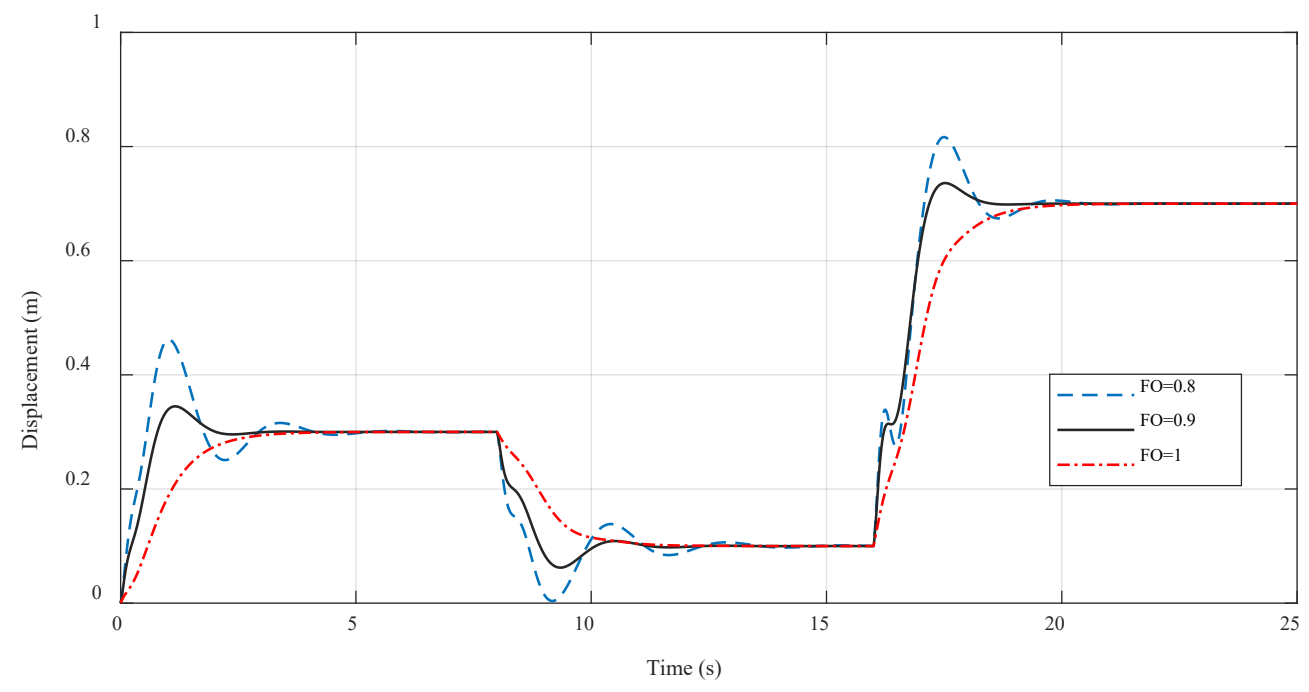

Fig. 2. Trolley motion.

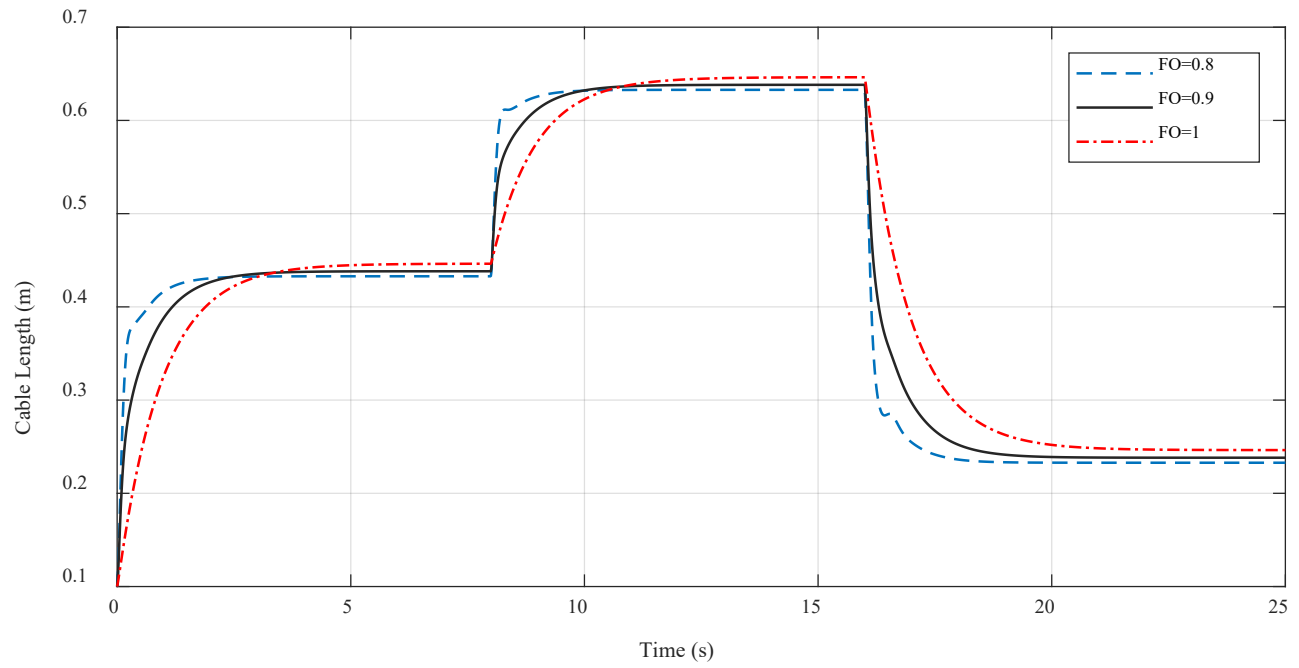

Fig. 3. Payload hoist.

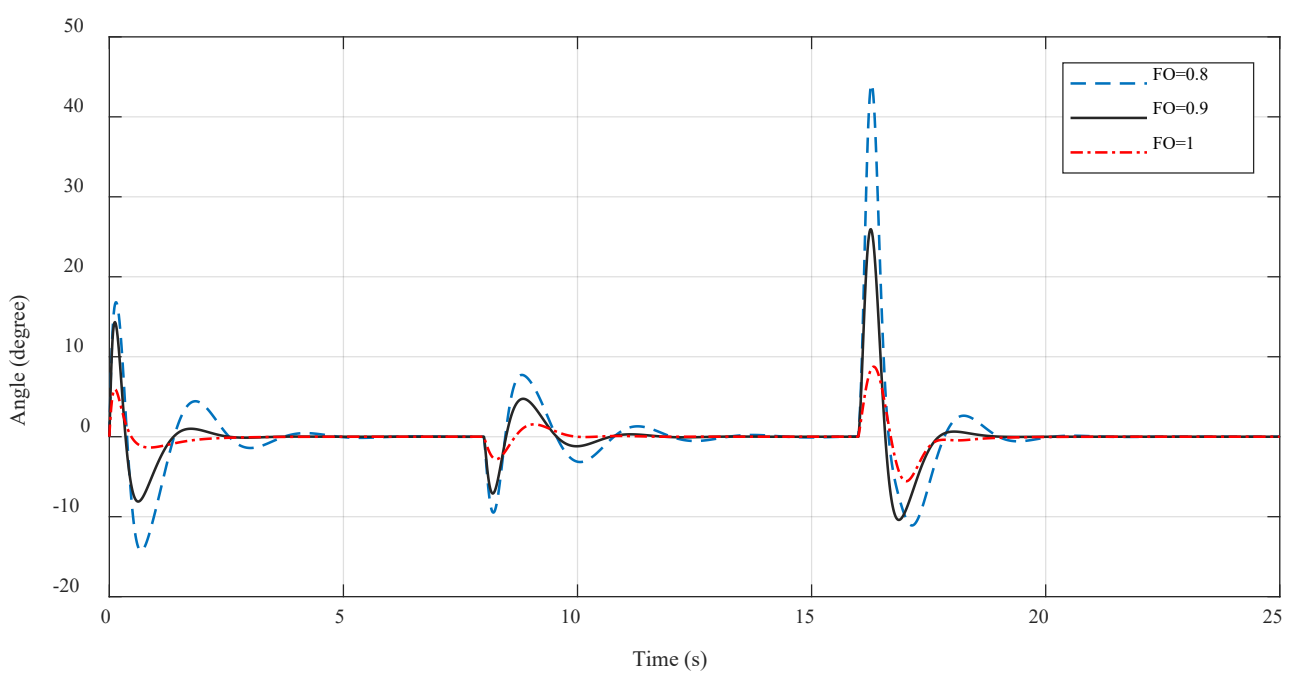

Fig. 4. Payload swing. 
JST: Smart Systems and Devices

Volume 31, Issue 1, May 2021, 068-075

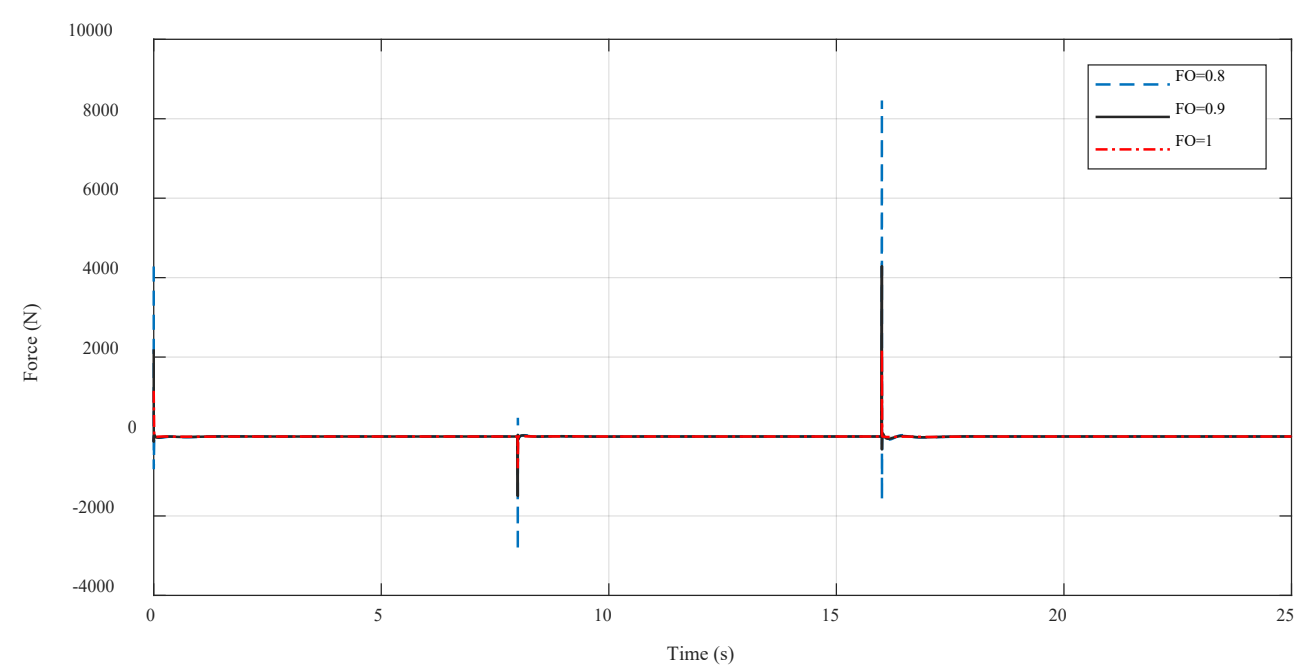

Fig. 5. Trolley-moving force.

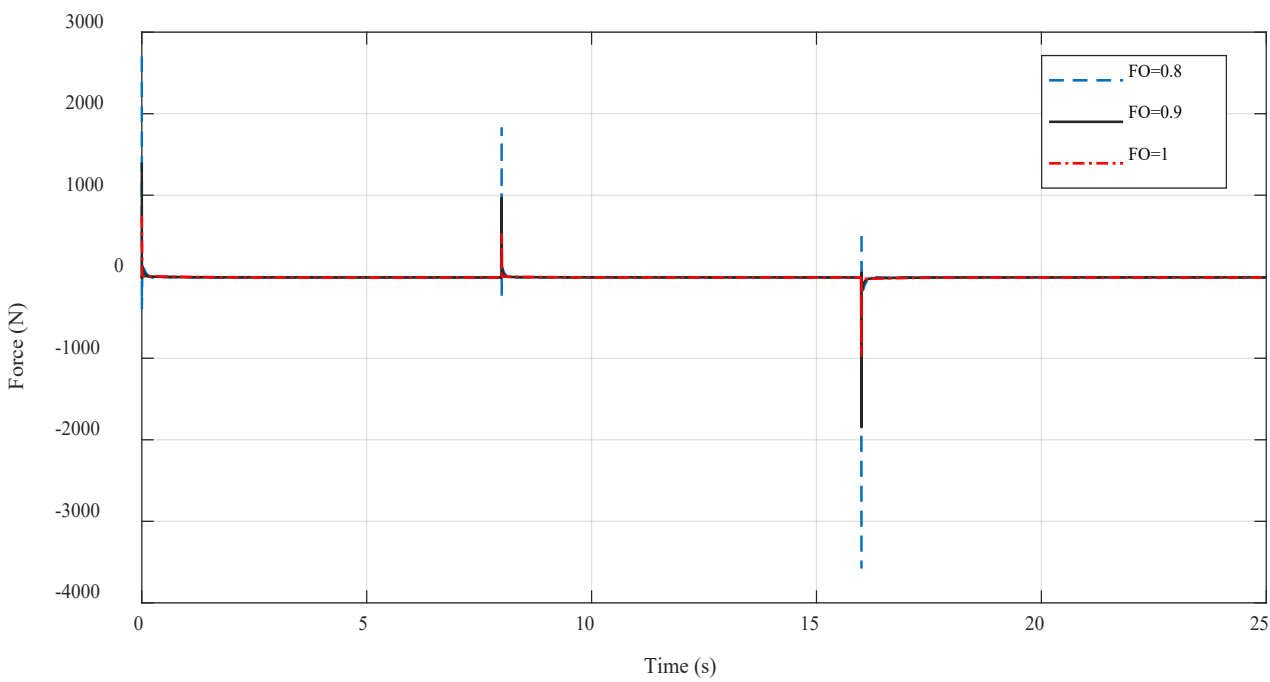

Fig. 6. Payload-hoisting force.

Proper selection of FO decides the control quality. Seen at Fig. 2, $\mathrm{FO}=0.8$ causes much oscillation of trolley motion, both $\mathrm{FO}=0.9$ and 1 assure the destination convergence, $\mathrm{FO}=0.9$ makes overshoot while $\mathrm{FO}=1$ does not. Varying $\mathrm{FO}$ from its origin $\mathrm{FO}=1$ can reduce settling time but causes the overshoots and even steady-state errors. Payload hoisting (Fig. 3) seems well for cases, however, convergence speed of $\mathrm{FO}=0.8$ is fastest. Payload swings are in small boundary (Fig. 4) at transient phase and absolutely suppressed at payload destinations. Depicted in Figs. 5 and 6, control inputs remain keen peaks due to high switched gains of controller. Generally, it is hard to say which FO is the best. Finding FO to achieve the optimal responses will be studied in the next article.
As a nature of SMC, the proposed controller assures the system robustness despite disturbance and uncertain environment. Considering the case $\mathrm{FO}=1$, we investigate the consistence of output responses when a crane faces parametric uncertainties. In fact, many crane parameters are variable and thus adjustable. A crane can lift and transfer the payload with various mass $m_{c}$ and volume. The frictions characterized by $b_{t}$ and $b_{r}$ are varied up to environment, temperature, and humidity of operation area. We consider the variation of three abovementioned parameters with two following cases:

Case 1: $\left[\Delta m_{c} \Delta b_{t} \Delta b_{r}\right]=\left[\begin{array}{lll}100 & 20-10\end{array}\right] \%$.

Case 2: $\left[\Delta m_{c} \Delta b_{t} \Delta b_{r}\right]=\left[\begin{array}{lll}-50-40 & 30\end{array}\right] \%$. 
JST: Smart Systems and Devices

Volume 31, Issue 1, May 2021, 068-075

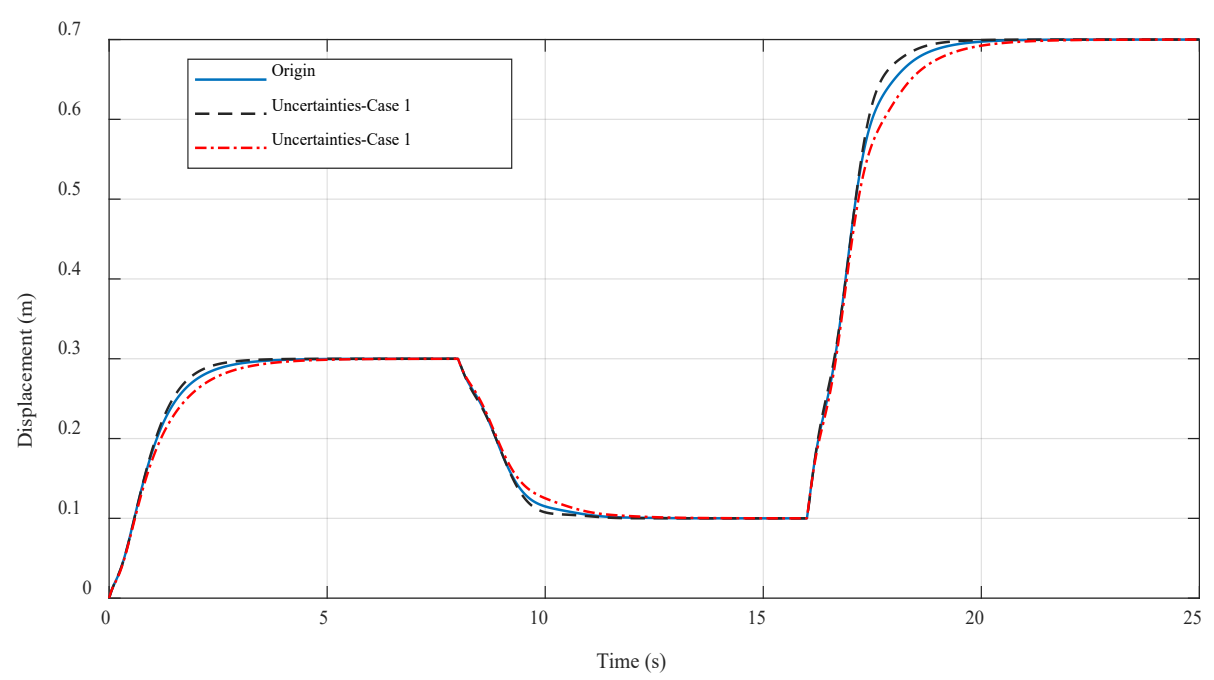

Fig. 7. Robustness of trolley motion.

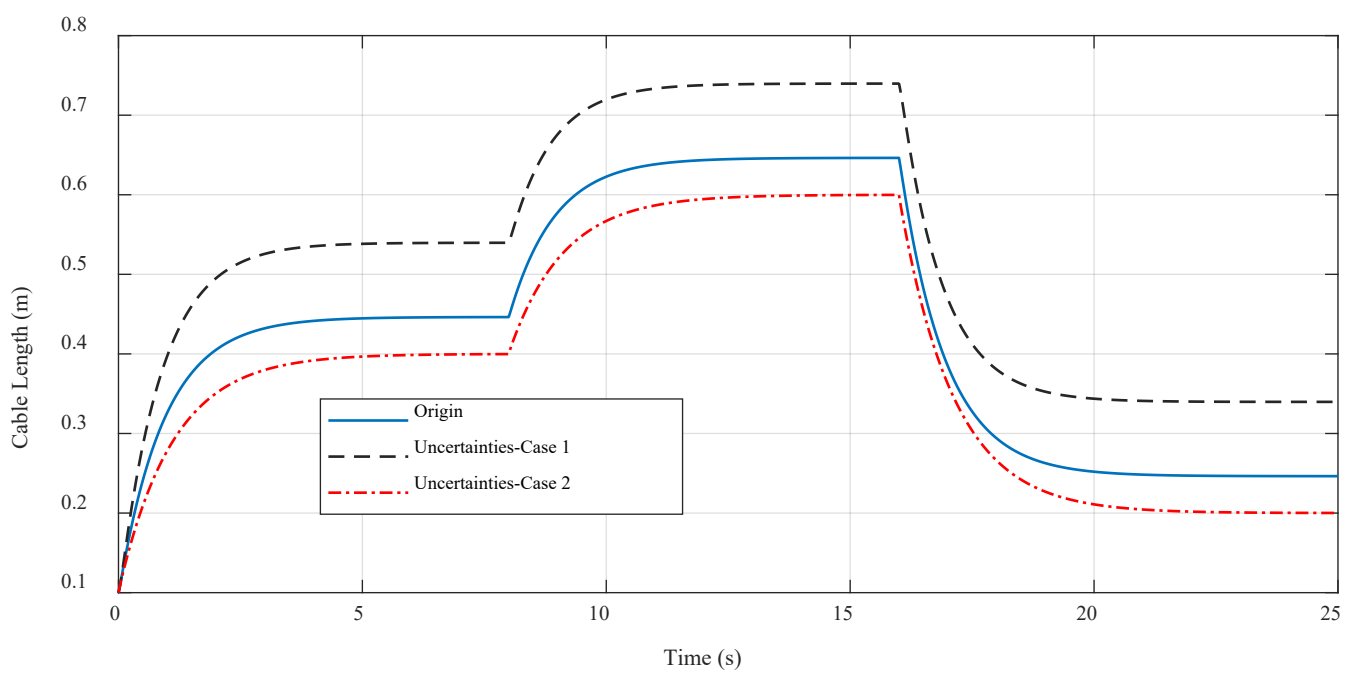

Fig. 8. Robustness of payload-hoist.

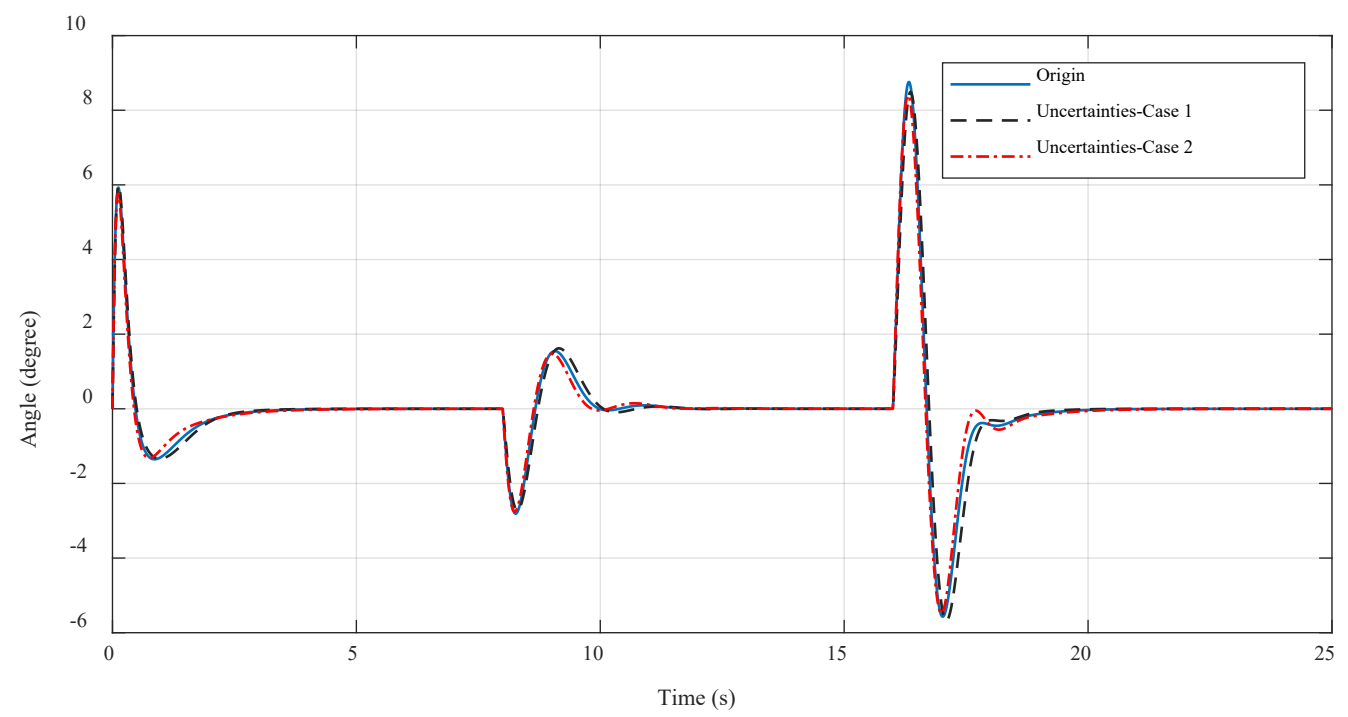

Fig. 9. Robustness of payload swing. 
JST: Smart Systems and Devices

Volume 31, Issue 1, May 2021, 068-075

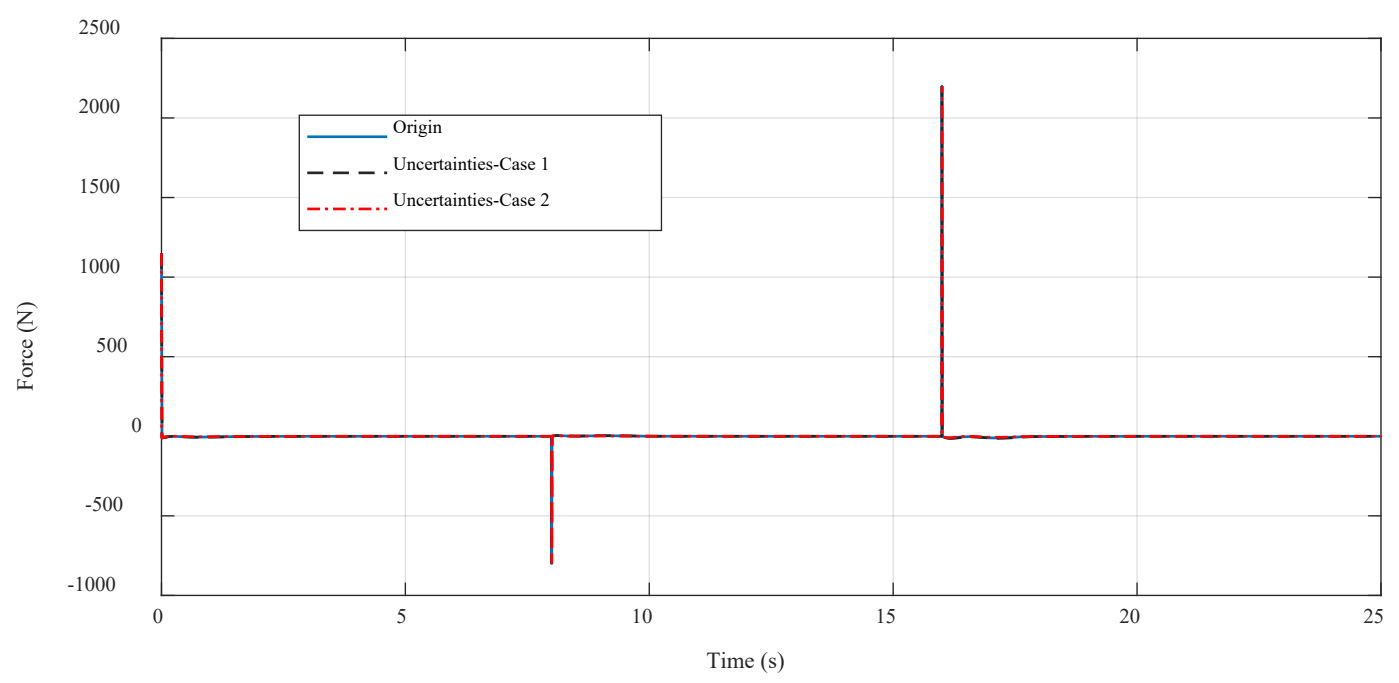

Fig. 10. Robustness of trolley-pushing force.

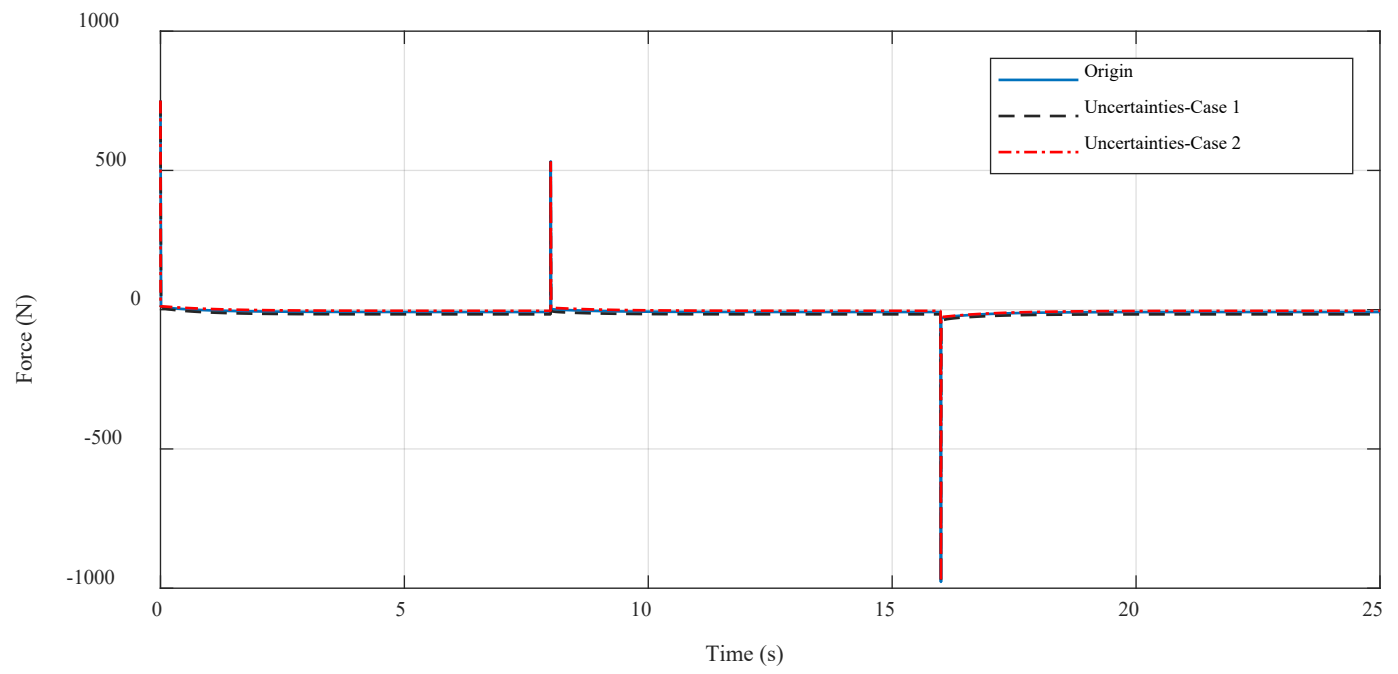

Fig. 11. Robustness of payload-hoisting force.

The simulation results when the system is subject to two cases of parametric uncertainties in comparison with original case are depicted in Fig.7 to 11. Despite parametric variations, trolley motion (Fig. 7) and payload swing (Fig. 9) still approach destination precisely. Parametric uncertainties only impact on outputs at transient states in which it causes small derivation. Hoisting the payload (Fig. 8) seems sensitive with the variation of parameters. It induces much not only transient-state derivations but also steady-state errors. Increasing swished gains $\eta=\operatorname{diag}\left(\eta_{1}, \eta_{2}\right)$ will improve robust feature however cause much chattering. The way to prevent chattering was discussed in Section 4.

\section{Conclusion}

By integrating FD into SMC structure, we successfully created a robust controller for tracking overhead crane with simultaneous drive of three motions. The controller works well for three cases of
FO that are considered as flexible control gains. Trolley and payload lifting responses reach destinations precisely while well vanishing payload swing. Enhancing for 3D motion and integrating adaptive control approaches will be conducted in the future study.

\section{Acknowledgments}

This research is funded by Vietnam National Foundation for Science and Technology Development under grant number 107.01-2019.301.

\section{References}

[1] Y. Sakawa, H. Sano, Nonlinear model and linear robust control of overhead travelling cranes, Nonlinear Analysis, Theory, Methods \& Applications 30 (1997) 2197-2207.

https://doi.org/10.1016/S0362-546X(97)86041-5

[2] L.A. Tuan, S.-G. Lee, V.-H. Dang, S. Moon, B.S. Kim, Partial feedback linearization control of a three- 
dimensional overhead crane, International Journal of Control, Automation and Systems 11 (2013) 718-727. https://doi.org/10.1007/s12555-012-9305-z

[3] A.C. Lecours, S. Foucault, T. Laliberté, B. Mayer-StOnge, C. Gosselin, A cable-suspended intelligent crane assist device for the intuitive manipulation of large payloads, IEEE/ASME Transactions on Mechatronics 21 (2016) 2073-2084. https://doi.org/10.1109/TMECH.2016.2531626

[4] A.Z. Al-Garni, K.A.F. Moustafa, and S.S.A.K. Javeed Nizami, Optimal control of overhead cranes," Control Engineering Practice 3 (1995) 1277-1284. https://doi.org/10.1016/0967-0661(95)00126-F

[5] X. Wu, X. He, Nonlinear energy-based regulation control of three-dimensional overhead cranes, IEEE Transactions on Automation Science and Engineering 14 (2017) 1297-1038.

https://doi.org/10.1109/TASE.2016.2542105

[6] H. Chen, Y. Fang, N. Sun, A swing constraint guaranteed mpc algorithm for underactuated overhead cranes, IEEE/ASME Transactions on Mechatronics 21 (2016) 2543-2555. https://doi.org/10.1109/TMECH.2016.2558202

[7] Y.J. Hua, Y.K. Shine, Adaptive coupling control for overhead crane systems, Mechatronics 17 (2007) 143152

https://doi.org/10.1016/j.mechatronics.2006.08.004

[8] L.A. Tuan, S.-G. Lee, L.C. Nho, D.-H. Kim, Model reference adaptive sliding mode control for three dimensional overhead cranes, International Journal of Precision Engineering and Manufacturing 14 (2013) 1329-1338. https://doi.org/10.1007/s12541-013-0180-1

[9] G Bartolini, A. Levant, A. Pisano, E. Usai, Adaptive second-order sliding mode control with uncertainty compensation, International Journal of Control 89 (2016) 1747-1758. https://doi.org/10.1080/00207179.2016.1142616

[10] D. Chwa, Sliding-mode-control-based robust finitetime antisway tracking control of 3-D overhead cranes, IEEE Transactions on Industrial Electronics 64 (2017) 6775-6784

https://doi.org/10.1109/TIE.2017.2701760

[11] X. Li, W. Yu, Anti-swing control for an overhead crane with fuzzy compensation, Intelligent Automation \& Soft Computing 18 (2012) 1-11. https://doi.org/10.1080/10798587.2012.10643223

[12] L.-H. Lee, P.-H. Huang, Y.-C. Shih, T.-C. Chiang, C.-Y. Chang, Parallel neural network combined with sliding mode control in overhead crane control system, Journal of Vibration and Control 20 (2014) 749-760.

https://doi.org/10.1177/1077546312464681

[13] N.B. Almutairi, M. Zribi. Sliding mode control of a three-dimensional overhead crane, Journal of Vibration and Control 15 (2009) 1679-1730. https://doi.org/10.1177/1077546309105095

[14] Z. Zhang, Y. Wu, Disturbance-observer-based antiswing control of underactuated crane systems via terminal sliding mode, IET Control Theory \& Applications 12 (2018) 2588-2594. https://doi.org/10.1049/iet-cta.2018.5344

[15] D.P. Nam, N.D. Phuoc, N.T.V Huong, Adaptive robust ability of high order sliding mode control for a 3 -D overhead crane system, Advances in Information and Communication Technology, Springer, (2017). https://doi.org/10.1007/978-3-319-49073-1_14

[16] Q.H. Ngo, N.P. Nguyen, C.N. Nguyen, T.H. Tran, Q.P Ha, Fuzzy sliding mode control of an offshore container crane, Ocean Eng. 140 (2017) 125-134. https://doi.org/10.1016/j.oceaneng.2017.05.019

[17] S. Frikha, M. Djemel, N. Derbel, A new adaptive neuro-sliding mode control for gantry crane, Int. J. Control Autom. Syst. 16 (2018) 559-565. https://doi.org/10.1007/s12555-017-0070-x

[18] G. Kim, K. Hong, Adaptive sliding-mode control of an offshore container crane with unknown disturbances, IEEE-ASME Trans. Mechatron. 24 (2019) 2850-2861. https://doi.org/10.1109/TMECH.2019.2946083

[19] I. Petráš (Ed.), Handbook of Fractional Calculus with Applications, vol. 6: Applications in Control, De Gruyter, (2019).

https://doi.org/10.1515/9783110571745

[20] M.A. Duarte-Mermoud, N. Aguila-Camacho, J.A. Gallegos, R. Castro-Linares, Using general quadratic Lyapunov functions to prove Lyapunov uniform stability for fractional order systems, Commun. Nonlinear Sci. Numer. Simul. 22 (2015) 650-659. https://doi.org/10.1016/j.cnsns.2014.10.008

[21] Y. Li, Y.Q. Chen, I. Podlubny, Mittag-Leffler stability of fractional order nonlinear dynamic systems. Automatica 45 (2009) 1965-1969. https://doi.org/10.1016/j.automatica.2009.04.003

[22] D. Matignon, Stability properties for generalized fractional differential systems, Proc. of Fractional Differential Systems: Models, Methods and App. 5 (1998) 145-158. https://doi.org/10.1051/proc:1998004 